\title{
Current and Future Targets for Mucosal Healing in Inflammatory Bowel Disease
}

\author{
Raja Atreya Markus F. Neurath \\ Medical Clinic 1, Friedrich-Alexander-University Erlangen-Nürnberg, Erlangen, Germany
}

\section{Keywords}

Mucosal healing - Inflammatory bowel diseases ·

Biologicals · Oligonucleotides · Small molecules

\section{Summary}

The induction and subsequent maintenance of mucosal healing has emerged as one of the central therapeutic goals in the management of patients with inflammatory bowel disease (IBD) (Crohn's disease and ulcerative colitis). Current and novel treatment options are assessed regarding their therapeutic efficacy on the basis of their ability to induce mucosal healing. However, there is still substantial debate about the precise definition of mucosal healing. Here, we will give an overview regarding the definitions of mucosal healing as well as its probable effects on long-term disease behavior and finally focus on current and potential therapeutic targets to achieve this therapeutic goal in IBD patients.

(c) 2017 S. Karger GmbH, Freiburg

\section{Introduction}

Inflammatory bowel diseases (IBD) comprise ulcerative colitis and Crohn's disease, which are characterized by idiopathic inflammation of the gastrointestinal tract. These disorders are marked by intermittent disease flares and sometimes chronic inflammation which result in irreversible mucosal damage, disability, and heightened incidence of colitis-associated neoplasias. The available therapeutic options for the treatment of IBD were rather limited for a long time, as the precise etiopathology of these diseases is not yet fully understood. Current concepts are based on the composition of genetic predisposition, environmental factors, and the intestinal microbiome that lead to an excessive activation of the mucosal immune system and subsequent inflammation. Due to the unclear etiological background, corticosteroids and broad-spectrum immunosuppressives are still part of the applied therapeutic algorithms [1-3]. Nevertheless, growing insights into the immunopathogenesis has enabled the emergence of several novel classes of therapies with different mechanisms of action [4]. The introduction of anti-tumor necrosis factor (TNF) agents marked the beginning of pathway-based treatments and initiated a new era of targeted therapies. The approval of therapies targeting the anti-adhesion mechanism or the proinflammatory cytokines IL(interleukin)-12/IL-23 represent novel promising developments in this regard. Despite the proven success of these antibody-based therapies, there is still an unmet clinical need as a substantial subgroup of patients fails to show clinical response to the available treatment options while another substantial proportion of initial responders demonstrates subsequent loss of response. At present, the rational management of these patients is therefore still a significant clinical challenge. The identification of novel targets amenable to pharmacological intervention offers promising concepts for future IBD therapy resulting in better patient care [4].

Clinical application of immunosuppressive and then biological agents enabled the physician to not only aim for clinical improvement but also raised hopes to ideally modulate the natural course of disease, preventing debilitating complications and preserving quality of life. These aims are reflected by key therapeutic goals that have shifted away from just focusing on the resolution of clinical symptoms and rather strive to attain better outcomes of the treated IBD patients. The induction and subsequent maintenance of mucosal healing has emerged as one of the central therapeutic goals as it has indirectly been associated with better disease control [5]. However, there is still substantial debate regarding the definition of mucosal healing. Nevertheless, current and novel treatment options are evaluated regarding their therapeutic efficacy on the basis of their ability to induce mucosal healing. In the following, we will give an overview on the definitions of mucosal healing, its probable effects on long-term disease behavior, and current and potential therapeutic targets to achieve this therapeutic goal in IBD patients.

\section{KARGER}

() 2017 S. Karger GmbH, Freiburg

Fax +497614520714 


\section{Mucosal Healing}

The assessment and monitoring of disease activity is indispensable for the optimal therapeutic management of IBD patients. Our therapeutic decisions are based on its evaluation, and analysis of disease activity is essential for endpoints in clinical trials. The mere improvement of clinical symptoms alone is not regarded as sufficient anymore, but must be ideally accompanied by mucosal restitution. This process has been termed mucosal healing, and it was proposed that it might lead to the modification of the natural disease course by slowing down or even preventing the progression of disease [5-7]. Mucosal healing is predominantly defined by endoscopic assessment of intestinal inflammation and is mostly referred to as the absence of mucosal ulcerations in Crohn's disease [8]. In ulcerative colitis, there is more disparity in the published definitions; however, an international consensus defined it as the absence of friability, blood, erosions, and ulcers of the gut mucosa (fig. 1) [9]. In order to evaluate the presence or absence of mucosal healing on endoscopy, various endoscopic scoring systems have been developed. The most frequently used endoscopic activity indices in Crohn's disease are the Crohn's Disease Endoscopic Index of Severity (CDEIS), the Simple Endoscopic Score for Crohn's Disease, and the Rutgeerts score. In ulcerative colitis, the Mayo Clinic endoscopic subscore and the Ulcerative Colitis Endoscopic Index of Severity (UCEIS) are those most widely used $[5,6]$. These indices allow us to determine improvements of endoscopic lesions, even when the rather rigid endpoint of mucosal healing and thereby the total disappearance of all mucosal ulcerations is not met. Other measures of assessing the disease activity are represented by the biomarkers C-reactive protein and fecal calprotectin. They are currently not regarded as targets for treatment but are helpful indices in monitoring the patient. Histopathology is another measure of inflammation but can also not be defined as a therapeutic target at the moment [10]. However, several recent studies suggest that histologic healing of intestinal inflammation is a better predictor of long-term patient outcomes in ulcerative colitis as compared to mucosal healing on endoscopy.

Mucosal healing can only represent an indispensable treatment goal if it serves as a validated surrogate marker for effective control of the disease and subsequently for the positive modulation of the disease course. Until now, there have been no long-term interventional studies comparing the outcome of clinical versus mucosal healing-based approaches for the disease course of IBD patients. There is no prospective evidence that mucosal healing, for example, predicts a reduced need for surgeries as an endpoint for disease modification. In contrast, there are substantial indirect indications from large case series that mucosal healing is associated with better patient-related outcome matters such as reduced risk of relapse, better steroid tapering, fewer hospitalization and surgeries, lower rate of colectomies, and improved ability to work $[5,6]$. These are important correlative findings, as the selected endpoints define improved quality of life for the IBD patient. Nevertheless, some aspects have to be taken into account before defining mucosal healing as an obtainable treatment goal in all IBD patients.

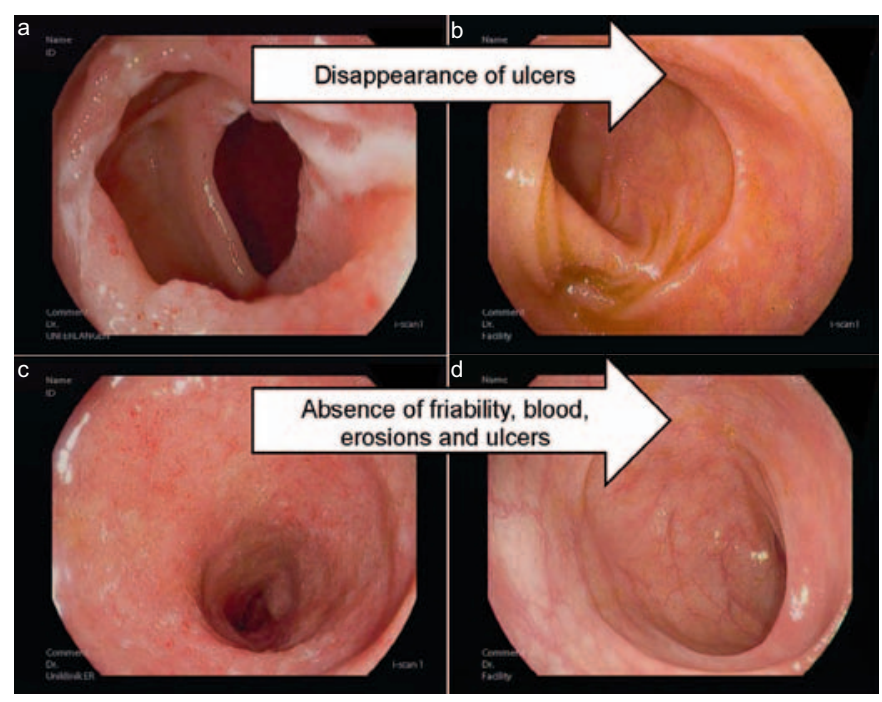

Fig. 1. Endoscopic images of a Crohn's disease patient a with mucosal ulcerations in the terminal ileum and $\mathbf{b}$ subsequent mucosal healing as defined by absence of ulcers and CDEIS scoring. Endoscopic images of an ulcerative colitis patient $\mathbf{c}$ with inflammation in the sigmoid and $\mathbf{d}$ subsequent mucosal healing as defined by absence of friability, blood, erosions, and ulcers as well as reduction of the endoscopic Mayo score.

In this regard, one has to consider that in some patients, especially those with long-standing Crohn's disease, mucosal healing may not always reflect healing of all layers of the tissue, as endoscopy only addresses mucosal rather than transmural healing. Furthermore, one has to consider safety aspects when trying to achieve mucosal healing by intensifying the ongoing therapy, thereby increasing the risk of potentially severe side-effects. Additionally, cost aspects associated with the adaption of therapies to attain mucosal healing cannot be disregarded. Altogether, definite prospective proof that an intensification of the therapy to achieve mucosal healing is able to modulate the natural disease course is still missing. This aspect should be demonstrated in prospective trials investigating the course of disease, as it would only then be justified to escalate therapy in a patient with clinical remission to achieve mucosal healing. It should therefore be carefully evaluated in which individual circumstances mucosal healing may be proposed as the target for therapeutic interventions.

\section{Mucosal Healing in Light of Already Approved Therapies}

\section{5-Aminosalicylates}

The different representatives of the substance class of 5-aminosalicylates (5-ASA) have proven their efficacy in inducing mucosal healing in ulcerative colitis patients with mild-to-moderate disease. Among studies with oral 5-ASA formulations, the potential to induce mucosal healing was demonstrated by multimatrix (MMX) 
5-ASA in two randomized, controlled phase III trials. When combining the patient populations from these studies $(n=517)$, the 8 -week complete mucosal healing rate defined as a sigmoidoscopy score of 0 was evident in $32 \%$ in both the $2.4 \mathrm{~g}$ and the $4.8 \mathrm{~g}$ daily MMX 5-ASA group compared with $16 \%(27 / 171)$ in the placebo group $[11,12]$. Another randomized clinical trial demonstrated mucosal healing with delayed-release oral 5-ASA at week 6 in 80 and $68 \%$ of mild-to-moderate ulcerative colitis patients treated with 4.8 and $2.4 \mathrm{~g} /$ day, respectively, underlining the importance of adequate 5-ASA dosing in patients with an acute flare [13]. In contrast to ulcerative colitis, there is no clear evidence that 5-ASA may induce mucosal healing in Crohn's disease.

\section{Corticosteroids}

Corticosteroids are ineffective in inducing mucosal healing in patients with Crohn's disease. One small study indicated that prednisolone was able to induce mucosal healing in only $12 \%$ of active Crohn's disease patients after a treatment period of 4-7 weeks [14]. Another study with 8 postoperative Crohn's disease patients even failed to show any induction of mucosal healing upon 6-9 weeks of prednisolone treatment [15]. In ulcerative colitis, however, there is sufficient evidence that corticosteroids are able to induce mucosal healing. This was first proven in a seminal publication by Truelove and Witts [16] in 1955. The group was able to show that high doses of oral corticosteroids (100 mg daily) induced mucosal healing in 30 versus $10 \%$ of placebo-treated active ulcerative colitis patients within 6 weeks [16]. Other studies confirmed these findings. The application of budesonide foam 2 $\mathrm{mg}$ twice daily for 2 weeks and then once daily for 4 weeks was able to induce mucosal healing in $56 \%$ in comparison to $44 \%$ of placebo-treated patients with mild-to-moderate ulcerative proctitis or ulcerative proctosigmoiditis $(n=546)$ after 6 weeks of therapy in a recently published study [17]. A pooled analysis of two phase III studies with budesonide MMX in patients with mildly to moderately active ulcerative colitis demonstrated mucosal healing (UCDAI mucosal appearance score of 0 ) rates of $64 \%$ for the budesonide MMX $9 \mathrm{mg}$ group in comparison to $36 \%$ in the placebo group at week 8 [18]. Overall, these data indicate that to a certain extent, different corticosteroid formulations are able to induce mucosal healing in ulcerative colitis patients. Nevertheless, it is important to keep in mind that corticosteroids are not able to maintain mucosal healing in the long run.

\section{Immunomodulators}

There are a couple of studies that have shown mucosal healing effects of azathioprine in Crohn's disease. The SONIC trial, for instance, compared the efficacy of azathioprine and infliximab as monotherapy or combination therapy in patients with Crohn's disease $(n=109)$ who were naïve to thiopurines or anti-TNF antibodies. After 26 weeks, only $16 \%$ of azathioprine monotherapy-treated patients exhibited mucosal healing, underlining the rather slow onset of action of this substance class [19].

The probably limited capacity of methotrexate to induce mucosal healing in Crohn's disease was shown in small studies. One of them reported that $36 \%(5 / 14)$ of refractory Crohn's disease patients had mucosal healing after 12 weeks of treatment, whereas none of the 7 patients with ulcerative colitis did [20].

Azathioprine is well recognized for its capacity to induce mucosal healing in ulcerative colitis patients. In a larger study comparing the effects of azathioprine monotherapy against infliximab monotherapy or the combination of both, mucosal healing rates after 4 months of therapy were 37,55 , and $63 \%$, respectively [21]. Regarding methotrexate, a recent trial also indicated that endoscopic healing without steroids at week 16 was obtained in 21 of 60 (35.0\%) patients randomized to the methotrexate arm in comparison to 13 of $51(25.5 \%)$ patients randomized to the placebo arm in a cohort of steroid-dependent ulcerative colitis patients $(\mathrm{p}=0.28)$, where clinical efficacy could not be proven on a statistically significant level [22].

Altogether, these data indicate that methotrexate has a limited capacity to induce mucosal healing in IBD patients, although available data are rather limited. In contrast, azathioprine is able to induce mucosal healing in Crohn's disease as well as ulcerative colitis, although a delayed period of action must be taken into account.

\section{Calcineurin Inhibitors}

Data investigating the efficacy of calcineurin inhibitors to induce mucosal healing in patients with severe ulcerative colitis are scarce. Assessment after 8 days of cyclosporine treatment did not find a significant effect on mucosal healing, although another study demonstrated a higher efficacy in comparison to methylprednisolone by week 4 [5]. Tacrolimus was able to induce mucosal healing in $14 / 32(44 \%)$ in comparison to $4 / 30(13 \%)$ placebo-treated patients after 2 weeks of oral therapy in a multicenter study with steroid-refractory ulcerative colitis patients [23]. However, there was no statistically significant difference regarding clinical remission in both groups, thus questioning the short-term clinical benefit of the observed endoscopic findings.

\section{Anti-TNF Antibodies}

The United States Food and Drug Administration approved the first anti-TNF agent infliximab for the treatment of moderate-tosevere Crohn's disease patients in 1998. Since then, several other representatives of this substance class have entered the market and have shown efficacy not only in inducing but also in maintaining mucosal healing. Its main molecular mechanism of action is attributed to the induction of intestinal $\mathrm{T}$ cell apoptosis [24], and the mucosal membrane-bound TNF expression may serve as a predictive biomarker for response, as recently assessed by an in vivo molecular imaging study [25]. 
The first controlled trial to evaluate the mucosal healing properties of anti-TNF agents was the ACCENT 1 study where 13/45 (29\%) of infliximab-treated Crohn's disease patients had mucosal healing at week 10 compared to just $3 \%(1 / 29)$ of the placebo group. The scheduled maintenance therapy with infliximab every 8 weeks led to mucosal healing at week 54 in 16/36 (44\%) patients, compared to $4 / 22(18 \%)$ patients who were given episodic treatment [26]. Although only a subgroup of patients was followed up endoscopically for 1 year, these results were a clear indicator for the capabilities of anti-TNF antibodies to maintain mucosal healing. The SONIC trial confirmed these results and found mucosal healing in 28/93 (30\%) of infliximab-treated patients at week 26, compared to $18 / 109$ (17\%) patients on azathioprine and 37/107 (44\%) patients on combination therapy with azathioprine and infliximab [19]. Subsequently, mucosal healing was also for the first time defined as the primary endpoint of clinical studies. The effects of adalimumab were evaluated in the EXTEND trial $(n=135)$, where 27 and $24 \%$ of adalimumab-treated Crohn's disease patients reached mucosal healing at week 12 and 52, respectively, in comparison to 13 and $0 \%$ given placebo after adalimumab induction therapy [8]. In the open-label MUSIC trial, certolizumab pegol was assessed regarding its efficacy to improve endoscopic inflammation in active Crohn's disease patients $(n=89)$. At week 10, after fourfold application of certolizumab pegol, the mucosal healing rate was $4 \%$, and after 54 weeks it was $8 \%$ [27].

In ulcerative colitis, it could similarly be shown that anti-TNF antibodies are capable of inducing mucosal healing. In the ACT1 and ACT2 trials, infliximab showed respective rates of mucosal healing in $75 / 121(62 \%)$ and $73 / 121(60 \%)$ patients compared to $41 / 121(34 \%)$ and $38 / 123(31 \%)$ of placebo-treated patients after 8 weeks. Scheduled maintenance therapy with $5 \mathrm{mg} / \mathrm{kg}$ infliximab resulted in mucosal healing rates of $46 \%(55 / 121)$ in infliximab- versus $18 \%(2 / 121)$ in placebo-treated patients in the ACT1 trial at week 54 [28]. Adalimumab similarly proved mucosal healing capacities, as a combined analysis of the two ULTRA studies reported mucosal healing rates of $43 \%(n=470)$ in the adalimumab and of $33 \%$ in the placebo group $(n=468)$ at week 8 [29]. In the ULTRA-2 trial, mucosal healing at weeks 8 and 52 could be recorded in 41 and $25 \%$ of the adalimumab-treated patients in comparison to 32 and $15 \%$ in the placebo group, respectively [30]. The anti-TNF antibody golimumab was tested in the PURSUIT-SC trial, and mucosal healing was observed at week 6 in 42 and $45 \%$ in the 200/100 and the $400 / 200 \mathrm{mg}$ groups, respectively, in comparison to $29 \%$ of patients under placebo therapy [31]. In the PURSUIT-M trial, mucosal healing at both weeks 30 and 54 could be achieved by $42 \%$ ( 50 $\mathrm{mg}$ ) and $42 \%(100 \mathrm{mg})$ of the golimumab-treated patients as opposed to $27 \%$ with placebo [32].

\section{Vedolizumab}

The anti- $\alpha 4 ß 7$ integrin antibody vedolizumab was approved for the treatment of Crohn's disease patients in 2014; however, there are limited data available regarding its capacity to induce mucosal healing so far. Data from a retrospective cohort study with moderate-to-severe Crohn's disease patients $(\mathrm{n}=212)$ showed 12-month cumulative rates of mucosal healing in $63 \%$ of vedolizumab-treated Crohn's disease patients [33]. In ulcerative colitis, mucosal healing rates obtained in the GEMINI 1 trial at week 52 were achieved in 52 and $56 \%$ of the patients receiving vedolizumab every 8 or 4 weeks, respectively, as opposed to $20 \%$ of the placebo-treated patients [34].

\section{Anti-IL-12/23 Antibody}

The monoclonal antibody ustekinumab that targets the common p40 subunit of the IL- 12 and IL-23 cytokines was approved in the USA and many European countries for the treatment of moderate-to-severe Crohn's disease patients at the end of 2016. Endoscopic subanalyses of the UNITI and IM-UNITI trials indicate a mucosal healing rate of $9 \%$ in the ustekinumab- compared to $4 \%$ in the placebo-treated patient groups after one applied infusion at week 8. Maintenance data at week 44 with initial responders to ustekinumab therapy at week 8 showed a rate of $17 \%$ achieving mucosal healing in the group receiving ustekinumab every 8 weeks compared to $6 \%$ in those receiving ustekinumab every 12 weeks, as opposed to $4 \%$ in the placebo group [35].

\section{Mucosal Healing in Light of Future Targets}

\section{Oligonucleotides}

Oligonucleotides are emerging as a potentially novel class of drugs with a variety of therapeutic targets. One of the first members to be tested in IBD patients was alicaforsen, an antisense inhibitor of the intracellular adhesion molecule-1, which is expressed on endothelial cells and is involved in leukocyte trafficking into the inflamed mucosa. After failure for the treatment of Crohn's disease patients, alicaforsen was successfully tested as an enema formulation in an open-label study with ulcerative colitis patients $(n=15)$ for a treatment period of 6 weeks. The rate of remission as defined by complete mucosal healing was observed in 33\% of patients at the end of the treatment [36]. Another study from the same group found a mucosal healing rate of $24 \%$ in mild-to-moderate leftsided ulcerative colitis patients treated with $240 \mathrm{mg}$ alicaforsen compared to a healing rate of $17 \%$ in a 5-ASA enema comparator group after 6 weeks of therapy [37].

The idea of topically applying an oligonucleotide to the inflamed mucosa was also investigated in a randomized trial with the Toll-like receptor 9 agonist cobitolimod (formerly known as DIMS0150), which was topically administered during colonoscopy in two single doses at week 0 and 4 in patients with moderate-to-severe ulcerative colitis $(\mathrm{n}=131)$. The DNA-based oligonucleotide missed the primary endpoint of clinical remission at week 12 but showed statistically significant results in patient-reported outcome measures at earlier time points. While mucosal healing at 
week 4 was reached by $35 \%$ of cobitolimod- versus $19 \%$ of placebo-treated patients $(\mathrm{p}=0.09)$, clinical remission combined with mucosal healing was achieved by 21 versus $5 \%$ patients and symptomatic remission with mucosal healing by 21 versus $2 \%$ patients, respectively, at week 4 [28]. Subsequent trials will have to further evaluate the therapeutic efficacy of this substance. The most impressive results until now in the emerging field of oligonucleotides in IBD have been recorded in a phase II trial with mongersen, an antisense oligonucleotide that abrogates SMAD 7 expression, leading to restoration of immunosuppressive TGF- 31 signaling. In a phase II trial with moderate-to-severe Crohn's disease patients $(\mathrm{n}=160)$, mongersen was able to induce clinical remission for a minimum of 2 weeks after prior 2-week therapy in 65\% (160 mg) compared to $10 \%$ (placebo) of the patients [39]. Endoscopic evaluation in an ongoing phase III trial will have to assess in how far this rapid clinical improvement is mirrored by mucosal healing on endoscopy.

\section{Anti-Trafficking Therapies}

\section{Etrolizumab}

Etrolizumab is a monoclonal antibody selectively directed against the $\beta 7$ subunit of the $\alpha 4 \beta 7$ and $\alpha E ß 7$ integrins. It is therefore able to inhibit the interaction between $\alpha 4 \beta 7$ and its ligand mucosal addressin cell adhesion molecule-1 to prevent mucosal leukocyte migration and the $\alpha \mathrm{E} ß 7 / \mathrm{E}$-cadherin binding to inhibit intraepithelial leukocyte retention. This dual mechanism of action might offer a possible therapeutic benefit. In a recently concluded phase II induction study, the primary endpoint of clinical remission was observed in $21 \%(100 \mathrm{mg})$ and $10 \%(300 \mathrm{mg})$ in the etrolizumab group and in $0 \%$ of the placebo group at week 10 . In the exploratory analyses, $10 / 39(26 \%)$ patients in the group receiving etrolizumab $100 \mathrm{mg}$ and $8 / 39$ (21\%) of those receiving etrolizumab $300 \mathrm{mg}+420 \mathrm{mg}$ loading dose had mucosal healing compared to $6 / 41(15 \%)$ patients in the placebo group at week 10. Mucosal aEß7-integrin expression served as a biomarker for therapeutic response. Additional studies to explore the maintenance effects of this substance are currently underway [40].

\section{Ozanimod}

Ozanimod is a selective oral small-molecule inhibitor that modulates the sphingosine 1-phosphate (S1P) receptor, which is necessary for activated lymphocytes to leave the lymph nodes. It causes the internalization of the $\mathrm{S} 1 \mathrm{P}$ receptor on lymphocytes, thereby disabling them to leave the lymph node. In a phase II trial with moderately to severely active ulcerative colitis patients $(n=186)$, ozanimod was able to reach clinical remission at week 8 in 16\% (0.5 $\mathrm{mg})$ and $14 \%(1 \mathrm{mg})$ of patients compared to $6 \%$ of the patients in the placebo group. Mucosal healing at week 8 was achieved in $28 \%$ $(0.5 \mathrm{mg})$ and $34 \%(1 \mathrm{mg})$ of patients and at week 32 in $32 \%(0.5 \mathrm{mg})$ and $33 \%(1 \mathrm{mg})$ of patients compared to $12 \%$ each in the placebo group at week 8 and 32 . Phase III studies are already underway to further evaluate the potential of ozanimod [41].

\section{Anti-Cytokine Antibodies}

\section{Risankizumab}

The monoclonal antibody risankizumab, which is directed against the p19 subunit of IL-23, was tested in a phase II trial in patients with moderate-to-severe Crohn's disease. After 12 weeks, $24 \%$ (200 mg) and 37\% (600 mg) of risankizumab-treated patients achieved clinical remission, compared with $15 \%$ of those receiving placebo. Endoscopic remission was achieved by $15 \%$ (200 mg) and $20 \%(600 \mathrm{mg})$ of patients receiving risankizumab compared to $3 \%$ of the placebo-treated patients. Larger studies evaluating the effects of risankizumab in Crohn's disease as well as in ulcerative colitis will soon be initiated [42].

\section{Janus Kinase Inhibitors}

\section{Tofactitinib}

Janus kinase (JAK) inhibitors are oral small-molecule therapies that target the non-receptor tyrosine kinase family which facilitate cytokine-mediated signaling through activation of the signal transducers and activators of transcription (STAT) pathway. The blockade of the JAK-STAT pathway inhibits the downstream upregulation of proinflammatory cytokines. Tofacitinib inhibits JAK 1 and JAK3, as well as JAK2 at higher doses, and was initially tested in a phase II trial in moderate-to-severe ulcerative colitis patients. Clinical remission at week 10 was reached with 10 and $15 \mathrm{mg}$ tofacitinib or placebo in 48, 41 and 10\%, respectively, of the cases. Mucosal healing was seen in 30,27 , and $2 \%$, respectively. There was a dosedependent increase in both low-density and high-density lipoprotein cholesterol [44]. Phase II studies in Crohn's disease did not demonstrate similar benefits. Recently presented data from a phase III trial in patients with ulcerative colitis showed 31 and $28 \%$ mucosal healing with $10 \mathrm{mg}$ tofacitinib in two separate induction studies, as compared to 16 and $12 \%$ of the placebo-treated patients.

\section{Filgotinib}

Filgotinib, an oral selective JAK1 inhibitor, was tested in a phase II study with moderate-to-severe Crohn's disease patients $(\mathrm{n}=174)$. Clinical remission was reached by $47 \%(60 / 128)$ of patients receiving filgotinib $200 \mathrm{mg}$ per day compared to $23 \%$ (10/44) of placebotreated patients at week 10 . Rates of mucosal healing were 4 versus $2 \%$ in the filgotinib and placebo group, respectively, after 10 weeks. Further phase III studies will have to confirm these findings and evaluate the efficacy of filgotinib in maintenance settings [45].

\section{Conclusion}

Mucosal healing has clearly established itself as one of the important treatment goals in IBD patients. Its possible guidance in daily clinical practice, however, will only be possible if there are uniform definitions. Novel and effective therapies will commercially be available in the near future and hopefully broaden our 
therapeutic possibilities to achieve mucosal healing in IBD patients. Only the characterization of their molecular mechanism of action and thus the possible prediction of therapeutic response will allow us to apply the growing number of potent therapeutic substances in a rational way and to avoid unselective usage which would not result in better care of our IBD patients in the end.

\section{Disclosure Statement}

R.A. has served as an advisor for Abbvie, Ferring, InDex Pharmaceuticals, and Takeda. M.F.N. has served as an advisor for MSD, Abbvie, Takeda, Boehringer Ingelheim, Giuliani, Janssen, Genentech, and Pentax.

\section{Funding}

This research was supported by the Emerging Fields Initiative of the Friedrich-Alexander University Erlangen-Nürnberg.

\section{References}

1 Strober W, Fuss I, Mannon P: The fundamental basis of inflammatory bowel disease. J Clin Invest 2007;117: 514-521.

2 Danese S, Fiocchi C: Ulcerative colitis. New Engl J Med 2011;365:1713-1725.

3 Kaser A, Zeissig S, Blumberg RS: Inflammatory bowel disease. Annu Rev Immunol 2010;28:573-621.

4 Danese S: New therapies for inflammatory bowel disease: from the bench to the bedside. Gut 2012;61:918 932.

5 Neurath MF, Travis SP: Mucosal healing in inflammatory bowel diseases: a systematic review. Gut. 2012;61: 1619-1635.

6 Pineton de Chambrun G, Peyrin-Biroulet L, Lemann $\mathrm{M}$, Colombel JF: Clinical implications of mucosal healing for the management of IBD. Nat Rev Gastroenterol Hepatol 2010;7:15-29.

7 Neurath MF: New targets for mucosal healing and therapy in inflammatory bowel diseases. Mucosal Immunol 2014;7:6-19.

8 Rutgeerts P, Van Assche G, Sandborn WJ, Wolf DC, Geboes K, Colombel JF, Reinisch W; EXTEND Investigators, Kumar A, Lazar A, Camez A, Lomax KG, Pollack PF, D'Haens G: Adalimumab induces and maintains mucosal healing in patients with Crohn's disease: data from the EXTEND trial. Gastroenterology 2012; 142:1102-1111.

9 D'Haens G, Sandborn WJ, Feagan BG, Geboes K, Hanauer SB, Irvine EJ, Lémann M, Marteau P, Rutgeerts P, Schölmerich J, Sutherland LR: A review of activity indices and efficacy end points for clinical trials of medical therapy in adults with ulcerative colitis. Gastroenterology 2007;132:763-786.

10 Peyrin-Biroulet L, Sandborn W, Sands BE, et al: Selecting therapeutic targets in inflammatory bowel disease (STRIDE): determining therapeutic goals for treat-totarget. Am J Gastroenterol 2015;110:1324-1338.

11 Lichtenstein GR, Kamm MA, Boddu P, Gubergrits N Lyne A, Butler T, Lees K, Joseph RE, Sandborn WJ: Effect of once- or twice-daily MMX mesalamine (SPD476) for the induction of remission of mild to moderately active ulcerative colitis. Clin Gastroenterol Hepatol 2007; 5:95-102.

12 Sandborn WJ, Kamm MA, Lichtenstein GR, Lyne A, Butler T, Joseph RE: MMX Multi Matrix System mesalazine for the induction of remission in patients with mild-to-moderate ulcerative colitis: a combined analysis of two randomized, double-blind, placebo-controlled trials. Aliment Pharmacol Ther 2007;26:205215.
13 Lichtenstein GR, Ramsey D, Rubin DT: Randomised clinical trial: delayed-release oral mesalazine $4.8 \mathrm{~g} /$ day vs. $2.4 \mathrm{~g} /$ day in endoscopic mucosal healing - ASCEND I and II combined analysis. Aliment Pharmacol Ther 2011;33:672-678.

14 Modigliani R, Mary JY, Simon JF, Cortot A, Soule JC, Gendre JP, Rene E: Clinical, biological, and endoscopic picture of attacks of Crohn's disease. Evolution on prednisolone. Groupe d'Etude Thérapeutique des Affections Inflammatoires Digestives. Gastroenterology 1990;98:811-818.

15 Olaison G, Sjödahl R, Tagesson C: Glucocorticoid treatment in ileal Crohn's disease: relief of symptoms but not of endoscopically viewed inflammation. Gut 1990;31:325-328.

16 Truelove SC, Witts LJ: Cortisone in ulcerative colitis; final report on a therapeutic trial. Br Med J 1955;2: 1041-1048.

17 Sandborn WJ, Bosworth B, Zakko S, Gordon GL, Clemmons DR, Golden PL, Rolleri RL, Yu J, Barrett AC, Bortey E, Paterson C, Forbes WP: Budesonide foam induces remission in patients with mild to moderate ulcerative proctitis and ulcerative proctosigmoiditis. Gastroenterology 2015;148:740-750.

18 Sandborn WJ, Danese S, D’Haens G, Moro L, Jones R, Bagin R, Huang M, David Ballard E, Masure J, Travis $\mathrm{S}$ : Induction of clinical and colonoscopic remission of mild-to-moderate ulcerative colitis with budesonide MMX 9 mg: pooled analysis of two phase 3 studies. Aliment Pharmacol Ther 2015;41:409-418.

19 Colombel JF, Sandborn WJ, Reinisch W, Mantzaris GJ, Kornbluth A, Rachmilewitz D, Lichtiger S, D’Haens G, Diamond RH, Broussard DL, Tang KL, van der Woude CJ, Rutgeerts P; SONIC Study Group: Infliximab, azathioprine, or combination therapy for Crohn's disease. N Engl J Med 2010;362:1383-1395.

20 Kozarek RA, Patterson DJ, Gelfand MD, Botoman VA, Ball TJ, Wilske KR: Methotrexate induces clinical and histologic remission in patients with refractory inflammatory bowel disease. Ann Intern Med 1989;110:353356.

21 Panaccione R, Ghosh S, Middleton S, Márquez JR, Scott BB, Flint L, van Hoogstraten HJ, Chen AC, Zheng H, Danese S, Rutgeerts P: Combination therapy with infliximab and azathioprine is superior to monotherapy with either agent in ulcerative colitis. Gastroenterology 2014;146:392-400
22 Carbonnel F, Colombel JF, Filippi J, et al.; European Crohn's and Colitis Organisation.; Groupe d'Étude Thérapeutique des Affections Inflammatoires Digestives: Methotrexate is not superior to placebo for inducing steroid-free remission, but induces steroid-free clinical remission in a larger proportion of patients with ulcerative colitis. Gastroenterology 2016;150 380-388.e4

23 Ogata $\mathrm{H}$, Kato J, Hirai F, Hida N, Matsui T, Matsumoto T, Koyanagi K, Hibi T: Double-blind, placebocontrolled trial of oral tacrolimus (FK506) in the management of hospitalized patients with steroid-refractory ulcerative colitis. Inflamm Bowel Dis 2012;18: 803-808.

24 Atreya R, Zimmer M, Bartsch B, Waldner MJ, Atreya I, Neumann H, Hildner K, Hoffman A, Kiesslich R, Rink AD, Rau TT, Rose-John S, Kessler H, Schmidt J, Neurath MF: Antibodies against tumor necrosis factor (TNF) induce T-cell apoptosis in patients with inflammatory bowel diseases via TNF receptor 2 and intestinal CD14 ${ }^{+}$macrophages. Gastroenterology 2011;141: 2026-2038.

25 Atreya R, Neumann H, Neufert C, et al: In vivo imaging using fluorescent antibodies to tumor necrosis factor predicts therapeutic response in Crohn's disease. Nat Med 2014;20:313-318.

26 Hanauer SB, Feagan BG, Lichtenstein GR, Mayer LF, Schreiber S, Colombel JF, Rachmilewitz D, Wolf DC, Olson A, Bao W, Rutgeerts P; ACCENT I Study Group: Maintenance infliximab for Crohn's disease: the ACCENT I randomised trial. Lancet 2002;359: 1541-1549.

27 Hébuterne X, Lémann M, Bouhnik Y, Dewit O, Dupas JL, Mross M, D’Haens G, Mitchev K, Ernault É, Vermeire S, Brixi-Benmansour H, Moreels TG, Mary JY, Marteau P, Colombel JF: Endoscopic improvement of mucosal lesions in patients with moderate to severe ileocolonic Crohn's disease following treatment with certolizumab pegol. Gut 2013;62:201-208.

28 Colombel JF, Rutgeerts P, Reinisch W, Esser D, Wang Y Lang Y, Marano CW, Strauss R, Oddens BJ, Feagan BG Hanauer SB, Lichtenstein GR, Present D, Sands BE, Sandborn WJ: Early mucosal healing with infliximab is associated with improved long-term clinical outcomes in ulcerative colitis. Gastroenterology 2011;141:11941201.

29 Sandborn WJ, van Assche G, Reinisch W, Colombe JF, D’Haens G, Wolf DC, Kron M, Tighe MB, Lazar A, Thakkar RB: Adalimumab induces and maintains clinical remission in patients with moderate-to-severe ulcerative colitis. Gastroenterology 2012;142:257-265. e1-3. 
30 Reinisch W, Sandborn WJ, Hommes DW, D'Haens G Hanauer S, Schreiber S, Panaccione R, Fedorak RN, Tighe MB, Huang B, Kampman W, Lazar A, Thakkar $\mathrm{R}$ : Adalimumab for induction of clinical remission in moderately to severely active ulcerative colitis: results of a randomised controlled trial. Gut 2011;60:780-787.

31 Sandborn WJ, Feagan BG, Marano C, Zhang H, Strauss R, Johanns J, Adedokun OJ, Guzzo C, Colombel JF, Reinisch W, Gibson PR, Collins J, Järnerot G Hibi T, Rutgeerts P; PURSUIT-SC Study Group: Subcutaneous golimumab induces clinical response and remission in patients with moderate-to-severe ulcerative colitis. Gastroenterology 2014;146:85-95.

32 Sandborn WJ, Feagan BG, Marano C, Zhang H, Strauss R, Johanns J, Adedokun OJ, Guzzo C, Colombel JF, Reinisch W, Gibson PR, Collins J, Järnerot G, Rutgeerts P; PURSUIT-Maintenance Study Group: Subcutaneous golimumab maintains clinical response in patients with moderate-to-severe ulcerative colitis. Gastroenterology 2014;146:96-109.

33 Dulai PS, Singh S, Jiang X, Peerani F, Narula N, Chaudrey K, Whitehead D, Hudesman D, Lukin D, Swaminath A, Shmidt E, Wang S, Boland BS, Chang JT, Kane S, Siegel CA, Loftus EV, Sandborn WJ, Sands BE, Colombel JF: The real-world effectiveness and safety of vedolizumab for moderate-severe Crohn's disease: results from the US VICTORY Consortium. Am J Gastroenterol 2016;111:1147-1155.

34 Feagan BG, Rutgeerts P, Sands BE, Hanauer S, Colombel JF, Sandborn WJ, Van Assche G, Axler J, Kim HJ, Danese S, Fox I, Milch C, Sankoh S, Wyant T, Xu J, Parikh A; GEMINI 1 Study Group: Vedolizumab as induction and maintenance therapy for ulcerative colitis. N Engl J Med 2013;369:699-710.
35 Sands BE, Gasink C, Jacobstein D, Gao LL, Johanns J, Szapary P, Colombel J-F, Targan S, Ghosh S, Sandborn W: Efficacy and safety of dose adjustment and delayed response to ustekinumab in moderate-severe Crohn's disease patients: results from the IM-UNITI Maintenance Study (abstract). United European Gastroenterol J 2016;4:A5.

36 Miner PB Jr, Geary RS, Matson J, Chuang E, Xia S, Baker BF, Wedel MK: Bioavailability and therapeutic activity of alicaforsen (ISIS 2302) administered as a rectal retention enema to subjects with active ulcerative colitis. Aliment Pharmacol Ther 2006;23:1427-1434.

37 Miner PB Jr, Wedel MK, Xia S, Baker BF: Safety and efficacy of two dose formulations of alicaforsen enema compared with mesalazine enema for treatment of mild to moderate left-sided ulcerative colitis: a randomized, double-blind, active-controlled trial. Aliment Pharmacol Ther 2006;23:1403-1413.

38 Atreya R, Bloom S, Scaldaferri F, Gerardi V, Admyre C, Karlsson Å, Knittel T, Kowalski J, Lukas M, Löfberg R, Nancey S, Petryka R, Rydzewska G, Schnabel R, Seidler U, Neurath MF, Hawkey C: Clinical effects of a topically applied Toll-like receptor 9 agonist in active moderate-to-severe ulcerative colitis. J Crohns Colitis 2016;10:1294-1302.

39 Monteleone G, Neurath MF, Ardizzone S, et al: Mongersen, an oral SMAD7 antisense oligonucleotide, and Crohn's disease. N Engl J Med 2015;372:1104-1113

40 Vermeire S, O'Byrne S, Keir M, et al: Etrolizumab as induction therapy for ulcerative colitis: a randomised, controlled, phase 2 trial. Lancet 2014;384:309-318.
41 Sandborn WJ, Feagan BG, Wolf DC, D'Haens G, Vermeire S, Hanauer SB, Ghosh S, Smith H, Cravets M, Frohna PA, Aranda R, Gujrathi S, Olson A; TOUCHSTONE Study Group: Ozanimod induction and maintenance treatment for ulcerative colitis. N Engl J Med 2016;374:1754-1762.

42 Feagan BG, Sandborn WJ, Panes J, Ferrante M, Louis E, D'Haens G, Franchimont D, Kaser A, Dewit O, Seidler U, Kim K-J, Neurath MF, Scholl P, Visvanathan S, Padula S, Herichova I, Soaita A, Hall D, Böcher WO Efficacy and safety of induction therapy with the selective IL-23 inhibitor risankizumab (BI 655066), in patients with moderate-to-severe Crohn's disease: results of a randomized, double-blind, placebo-controlled phase II study. Digestive Disease Week, San Diego, USA, 21-24th May 2016. Abstract ID 2483687.

43 Atreya R, Mudter J, Finotto S, et al: Blockade of interleukin 6 trans signaling suppresses T-cell resistance against apoptosis in chronic intestinal inflammation: evidence in Crohn disease and experimental colitis in vivo. Nat Med 2000;6:583-588.

44 Sandborn WJ, Ghosh S, Panes J, Vranic I, Su C, Rousell S, Niezychowski W; Study A3921063 Investigators: Tofacitinib, an oral Janus kinase inhibitor, in active ulcerative colitis. N Engl J Med 2012;367:616-624

45 Vermeire S, Schreiber S, Petryka R, Kuehbacher T, Hebuterne X, Roblin X, Klopocka M, Goldis A, Wisniewska-Jarosinska M, Baranovsky A, Sike R, Stoyanova $\mathrm{K}$, Tasset C, Van der Aa A, Harrison P: Clinical remission in patients with moderate-to-severe Crohn's disease treated with filgotinib (the FITZROY study) results from a phase 2, double-blind, randomised, placebo-controlled trial. Lancet 2017;389:266-275. 